\title{
Notes on the Quantization of FRW Model in the Presence of a Cosmological Constant and Radiation
}

\author{
G. A. Monerat, E. V. Corrêa Silva, G. Oliveira-Neto, \\ Departamento de Matemática e Computação, Faculdade de Tecnologia, \\ Universidade do Estado do Rio de Janeiro, \\ Estrada Resende-Riachuelo, $s / n^{o}$, Morada da Colina \\ CEP 27523-000, Resende-RJ, Brazil. \\ L. G. Ferreira Filho, \\ Departamento de Mecânica e Energia, Faculdade de Tecnologia, \\ Universidade do Estado do Rio de Janeiro, \\ Estrada Resende-Riachuelo, $s / n^{o}$, Morada da Colina \\ CEP 27523-000, Resende-RJ, Brazil. \\ and N. A. Lemos \\ Instituto de Física, Universidade Federal Fluminense, \\ R. Gal. Milton Tavares de Souza $s / n^{o}$, Boa Viagem \\ CEP 24210-340, Niterói-RJ, Brazil \\ (Received on 7 October, 2005)
}

\begin{abstract}
In the present work, we use the formalism of quantum general relativity in order to quantize a FriedmannRobertson-Walker model in the presence of a negative cosmological constant and radiation. The model has spatial sections with positive constant curvature. The wave-function of the model satisfies a Wheeler-DeWitt equation, for the scale factor, which has the form of the Schrödinger's equation for the quartic anharmonic oscillator. We find the eigenvalues and eigenfunctions by using a method first developed by Chhajlany and Malnev. After that, we use the eigenfunctions in order to construct wave-packets for evaluating the time-dependent, expected value of the scale factor. We find that, the expected value of the scale factor oscillates between maximum and minimum values. Since the scale factor never vanishes, we conclude that the model does not have a singularity.
\end{abstract}

One of the motivations for the quantization of cosmological models was that of avoiding the initial Big Bang singularity. Since the pioneering work in quantum cosmology due to DeWitt [1], workers in this field have been attempting to prove that quantum cosmological models entail only regular space-times. An important contribution to this issue was given by Hartle and Hawking [2], who proposed the no-boundary boundary condition, which selects only regular space-times to contribute to the wave-function of the Universe, derived in the path integral formalism. Therefore, by construction, the no-boundary wave-functions are everywhere regular and predict a non-singular initial state for the Universe. Using that boundary condition, in certain particular cases the noboundary wave-function can be explicitly computed [2-4]. Another way by which one may compute the wave-function of the Universe is by directly solving the Wheeler-DeWitt equation [1]. The wave-function of the Universe for some important models have been computed using this approach [5-10].

Several important theoretical results and predictions in quantum cosmology have been obtained with a negative cosmological constant [11], [12] and [3]. Besides that, we think it is important to understand more about such models which represent bound Universes (analogous to uni-dimensional atoms, in the present situation).

In the present paper, we use the formalism of quantum cosmology in order to quantize a Friedmann-Robertson-Walker model in the presence of a negative cosmological constant and radiation. The radiation is treated by means of the vari- ational formalism developed by Schutz [13]. The model has spatial sections with positive constant curvature. The wavefunction of the model satisfies a Wheeler-DeWitt equation, for the scale factor, which has the form of the Schrödinger's equation for the quartic anharmonic oscillator. We find the eigenvalues and eigenfunctions by using a method first developed by Chhajlany and Malnev. After that, we use the eigenfunctions in order to construct wave-packets for evaluating the time-dependent, expected value of the scale factor. We find that the expectation value of the scale factor shows bounded oscillations and since it never vanishes, we conclude that the model does not have a singularity.

Friedmann-Robertson-Walker cosmological models are characterized by the scale factor $a(t)$ and have the following line element,

$$
d s^{2}=-N(t)^{2} d t^{2}+a(t)^{2}\left(\frac{d r^{2}}{1-k r^{2}}+r^{2} d \Omega^{2}\right),
$$

where $d \Omega^{2}$ is the line element of the two-dimensional sphere with unitary radius, $N(t)$ is the lapse function and $k=1$. It means that the constant curvature of the spatial sections is positive. The spatial sections may be thought as $S^{3}$ 's. We are using the natural unit system, where $\hbar=c=G=1$. The matter content of the model is represented by a perfect fluid with four-velocity $U^{\mu}=\delta_{0}^{\mu}$ in the comoving coordinate system used, plus a negative cosmological constant. The total energy-momentum tensor is given by, 


$$
T_{\mu, v}=(\rho+p) U_{\mu} U_{v}-p g_{\mu, \nu}-\Lambda g_{\mu, \nu},
$$

where $\rho$ and $p$ are the energy density and pressure of the fluid, respectively. Here, we assume that $p=\rho / 3$, which is the equation of state for radiation. This choice may be considered as a first approximation to treat the initial content of the Universe and it was made as a matter of simplicity. It is clear that a more complete treatment should describe the radiation, present in the primordial Universe, in terms of the electromagnetic field. Einstein's equations for the metric (1) and the energy momentum tensor (2) are equivalent to the Hamilton equations generated by the super-hamiltonian constraint

$$
\mathcal{H}=-\frac{p_{a}^{2}}{12}-3 a^{2}+\Lambda a^{4}+p_{T}
$$

where $p_{a}$ and $p_{T}$ are the momenta canonically conjugated to $a$ and $T$ the latter being the canonical variable associated to the fluid [10].

In the case of the model studied here, the scale factor performs bounded oscillations. When the scale factor vanishes we have the formation of a singularity which may be either a Big Bang or a Big Crunch.

Using the Dirac formalism for quantizing constrained systems [14], we obtain from $\mathcal{H}$ Eq. (3) the following WheelerDeWitt equation,

$$
\left(\frac{1}{12} \frac{\partial^{2}}{\partial a^{2}}-3 a^{2}+\Lambda a^{4}\right) \Psi(a, \tau)=-i \frac{\partial}{\partial \tau} \Psi(a, \tau),
$$

where $\Psi(a, \tau)$ is the wave-function of the model and the new variable $\tau=-T$ has been introduced.

The operator $\hat{\mathcal{H}}$ is self-adjoint [8] with respect to the internal product,

$$
(\Psi, \Phi)=\int_{0}^{\infty} d a \Psi(a, \tau)^{*} \Phi(a, \tau)
$$

if the wave functions are restricted to the set of those satisfying either $\Psi(0, \tau)=0$ or $\Psi^{\prime}(0, \tau)=0$, where the prime / means the partial derivative with respect to $a$.

The Wheeler-DeWitt equation (4) is the Schrödinger equation for the quartic anharmonic oscillator and may be solved by writing $\Psi(a, \tau)$ as

$$
\Psi(a, \tau)=e^{-i E \tau} \eta(a)
$$

where $\eta(a)$ depends solely on $a$. Then $\eta(a)$ satisfies the eigenvalue equation

$$
-\frac{d^{2} \eta(a)}{d a^{2}}+V_{e}(a) \eta(a)=12 E \eta(a),
$$

where the effective potential $V_{e}(a)$ is given by

$$
V_{e}(a)=36 a^{2}-12 \Lambda a^{4} \text {. }
$$

The method of Chhajlany and Malnev [15] starts with the addition of an extra term to the original anharmonic oscillator potential, so that the modified Hamiltonian admits a subset of manifestly normalizable solutions. In the case we are considering, the extra term to be added to the effective potential (8) is proportional to $a^{6}$. In terms of that new enlarged potential, the eigenvalue equation (7) may be re-written as

$$
\eta^{\prime \prime}(a)+\left(\varepsilon-\alpha a^{2}-b a^{4}-c a^{6}\right) \eta(a)=0
$$

where $\varepsilon=12 E, \alpha=36, b=-12 \Lambda, c$ is a parameter to be determined by the method. The Ansatz for the solution of Eq. (9) takes the form

$$
\eta(a)=N \exp \left(-\frac{c}{4} a^{4}-\frac{\gamma}{2} a^{2}\right) v(a),
$$

and has finite norm for $c>0$. Here, $v(a)$ is a polynomial of a certain degree, yet to be chosen; the parameter $\gamma$ is to be chosen according to our convenience, as we shall see; $N$ is a normalization factor. The method is based on the fact, shown in Ref. [15], that the larger the degree of the polynomial $v(a)$, the smaller $c$ is. Therefore, if one increases the order of $v(a)$, the energy eigenvalues predicted by the present method tend monotonically, from above, to the energy eigenvalues of the original problem. One important property of the method is that the convergence is very fast. This means that one does not need to use a polynomial of very large order to obtain a good agreement with the energies of anharmonic oscillators already computed in the literature by other methods.

The next step is the substitution of the Ansatz (10) into the differential equation (9), which gives rise to an equation for the polynomial $v(a)$. Then, writing $v(a)=\sum_{n} \beta_{n} a^{n}$ and inserting it in that equation for the polynomial $v(a)$, along with the condition $2 c \gamma=b$ [15], we manage to find:

$$
(\varepsilon-\gamma) \beta_{0}+2 \beta_{2}=0, \quad(\varepsilon-3 \gamma) \beta_{1}+6 \beta_{3}=0,
$$

and the general recurrence relation for the polynomial coefficients $\beta_{n}$,

$$
\begin{aligned}
(n+4)(n+3) \beta_{n+4} & +[\varepsilon-\gamma(2 n+5)] \beta_{n+2} \\
+\left[\gamma^{2}-\alpha-c(2 n+3)\right] \beta_{n} & =0
\end{aligned}
$$

for $n \geq 0$. The degree of the polynomial $v(a)$ is fixed to be, say $K$, by imposing the following conditions in (12),

$$
\beta_{K} \neq 0, \quad \beta_{K+2}=\beta_{K+4}=0 .
$$

Due to the nature of the recurrence relation (12), it is clear that by fixing $K$ to be even (odd) the resulting polynomial $v(a)$ will be even (odd). Then, the coefficients $\beta_{n}, n=2,4, \ldots, K$ $(n=3,5, \ldots, K)$, will be determined in terms of $\beta_{0}\left(\beta_{1}\right)$ by the normalization condition. In the present situation, we restrict our attention to the case of an odd polynomial. It means that, $K=2 m+1$ for $m=0,1,2, \ldots$. This condition is imposed in order that our wave-function vanishes at $a=0$. 
Eqs. (12) and (13) require that the coefficient $\beta_{K}$ must vanish; then

$$
\gamma^{2}=\alpha+c(2 K+3) .
$$

Combining this with $2 c \gamma=b$, we obtain a cubic algebraic equation in the parameter $c$,

$$
4 c^{3}(2 K+3)+4 \alpha c^{2}-b^{2}=0 .
$$

The solutions of this equation depend on the known parameters $b$ and $K$. We must find the real, positive root to this equation so that the Ansatz Eq. (10) be normalizable. That real positive root, as proved in Ref. [15], is a monotonically decreasing function of $K$. Therefore, the greater the polynomial degree, the better the agreement between the energy eigenvalues obtained by this method and the actual energy eigenvalues.

Now, by setting the condition $\beta_{K+2}=0$ in Eq. (12) we may determine the corresponding $(m+1)$ energy eigenvalues $\varepsilon$ and polynomial coefficients $\beta_{n}$. With those coefficients $\beta_{n}$, we obtain the appropriate polynomial $v_{l}(a)$; the index $l=1,2, \ldots, m+1$ represents the energy level, for each of which we shall have an eigenfunction $\eta_{l}(a)$ and a wave-function $\Psi_{l}(a, \tau)=\exp \left(-i E_{l} \tau\right) \eta_{l}(a)$, according to Eqs. (6) and (10).

We construct a general solution to the Wheeler-DeWitt equation (4) by taking linear combinations of the $\Psi_{l}(a, \tau)$ 's,

$$
\Theta(a, \tau)=\sum_{l=1}^{m+1} A_{l}(E) \eta_{l}(a) e^{-i E_{l} \tau},
$$

as a matter of simplicity we shall set the coefficients $A_{l}(E)$ to one, in what follows.

With those combinations we compute the expected value for the scale factor $a$, following the many worlds interpretation of quantum mechanics [16]. In the present situation, we may write the expected value for the scale factor $a$ is

$$
\langle a\rangle(\tau)=\frac{\int_{0}^{\infty} a|\Theta(a, \tau)|^{2} d a}{\int_{0}^{\infty}|\Theta(a, \tau)|^{2} d a} .
$$

In order to obtain the results, we shall use the value of $\Lambda=-0.1$, therefore one has $b=1.2$ in Eq. (9). Also, we shall fix the polynomial degree to be $K=45$. It means that, we shall have 23 energy levels and 23 eigenfunctions $\eta_{l}(a)$. Using the values of $K$ and $b$, we solved Eq. (15) to find $c=0.090068960669615962974$. Now, we obtain the energy levels; they are listed in Table I. The first few lowest energy levels are in agreement with the ones computed, perturbatively, by Landau for the quartic anharmonic potential, equivalent to the present case [17]. For the present case, we may also compare the original potential Eq. (8) with the auxiliary potential,

$$
U(a)=36 a^{2}+1.2 a^{4}+0.008112417676 a^{6} .
$$

Then, we obtain a relative error $\varepsilon$ of less than $1 \%$ in the interval $a \in[0,2.728021504]$. Where $\varepsilon$ is given by,

$$
\varepsilon=\left|\frac{V(a)-U(a)}{V(a)}\right| \text {. }
$$

After that, we substitute the energies in the set of Eqs. (12) and compute the coefficients $\beta_{n}$. With these $\beta_{n}$, we write the following $\eta(a, \tau)$, according to Eq. (10),

$$
\begin{aligned}
& \eta_{l}(a)=N_{l} v_{l}(a) \times \\
& e^{-0.022517240167403990744 a^{4}-3.3307811899866029985 a^{2}},
\end{aligned}
$$

where

$$
v_{l}(a)=\sum_{i=0}^{22} A_{l, 2 i+1} a^{2 i+1} .
$$

The coefficients $N_{l}$ are normalization coefficients and $i=$ $0,1, \ldots, m$. The complete list of values for the $N_{l}$ 's and the $A_{l, 2 i+1}$ 's as well as the study of the cases where $k=0$ and $k=-1$ are given in reference [18].

Next, we construct the wave-packet $\Theta(a, \tau)$ with the aid of the $\eta_{l}(a)$, according to Eqs. (16), (20) and the energy levels in Table I. Finally, using the wave-packet $\Theta(a, \tau)$ we compute the expected value for the scale factor $a$, Eq. (17). The result is shown in Fig. 1; it can be seen that $\langle a\rangle$ does not vanish, therefore we may say that the quantization of this model removed the singularities it had at the classical level. It is clear from Fig. 1, also, that $\langle a\rangle$ performs bounded oscillations. That means that the spatial sections $S^{3}$ 's oscillate between finite maximum and minimum radius.

\begin{tabular}{|c|c|}
\hline Level & $k=1$ \\
\hline$E_{1}$ & 1.5103016760578712464 \\
\hline$E_{2}$ & 3.5509871014722954423 \\
\hline$E_{3}$ & 5.6230931685080087038 \\
\hline$E_{4}$ & 7.7256531719671366439 \\
\hline$E_{5}$ & 9.8577817762723638293 \\
\hline$E_{6}$ & 12.018664367453930157 \\
\hline$E_{7}$ & 14.207548216681935132 \\
\hline$E_{8}$ & 16.423735430300010131 \\
\hline$E_{9}$ & 18.666574187417793427 \\
\hline$E_{10}$ & 20.935469528987599984 \\
\hline$E_{11}$ & 23.229800589269486517 \\
\hline$E_{12}$ & 25.549220854858484858 \\
\hline$E_{13}$ & 27.892697492531834013 \\
\hline$E_{14}$ & 30.260978882469751228 \\
\hline$E_{15}$ & 32.651319599290796010 \\
\hline$E_{16}$ & 35.066840708164574204 \\
\hline$E_{17}$ & 37.502643477205645295 \\
\hline$E_{18}$ & 39.963027226267456788 \\
\hline$E_{19}$ & 42.443644402959303694 \\
\hline$E_{20}$ & 44.946892054208187300 \\
\hline$E_{21}$ & 47.470929938318337116 \\
\hline$E_{22}$ & 50.01608754545613080 \\
\hline$E_{23}$ & 52.581852999800707709 \\
\hline
\end{tabular}

TABLE I: The lowest calculated energy levels.

\section{Acknowledgments}

E. V. Corrêa Silva thanks CNPq for partial financial support. 


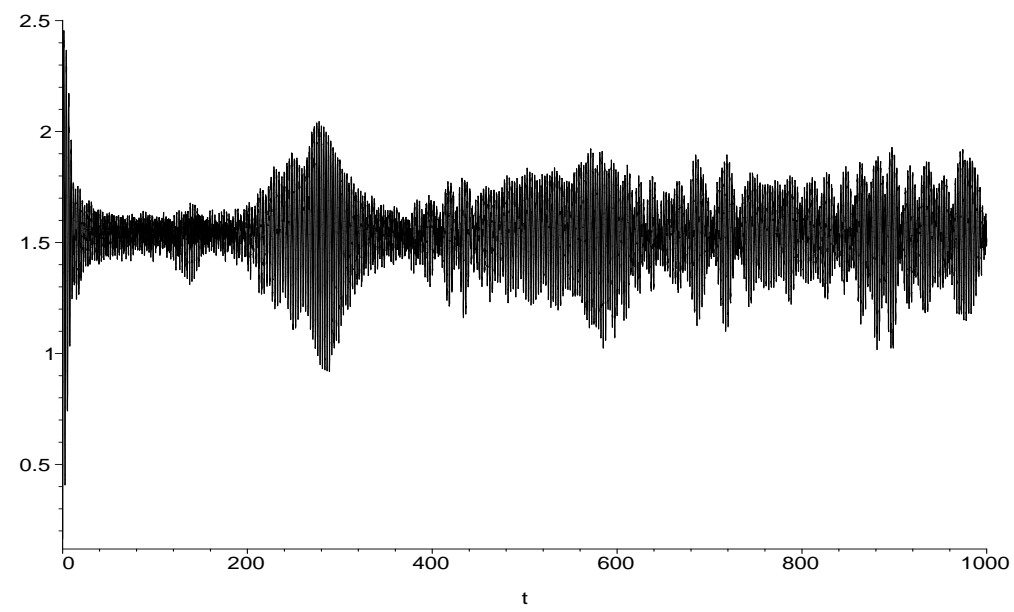

FIG. 1: Behavior of the expectation value of the scalar factor.

[1] B. S. DeWitt, Phys. Rev. D 160, 1113 (1967).

[2] J. B. Hartle and S. W. Hawking, Phys. Rev. D28, 2960 (1983).

[3] G. Oliveira-Neto, Phys. Rev. D 58, 10750 (1998);

[4] Y. Fujiwara et al., Class. Quantum Grav. 7163 (1992); Phys. Rev. D 44, 1756 (1991); J. Louko and P. J. Ruback, Class. Quantum Grav. 8, 91 (1991); J. J. Halliwell and J. Louko, Phys. Rev. D 42, 3997 (1990);

[5] Mariam Bouhmadi-Lopez and Paulo Vargas Moniz, Phys. Rev. D 71, 063521 (2005); I. G. Moss and W. A. Wright, Phys. Rev. D 29, 1067 (1984); M. J. Gotay and J. Demaret, Phys. Rev. D 28, 2402 (1983);

[6] O. Bertolami and J. M. Mourão, Class. Quantum Grav. 8, 1271 (1991).

[7] M. Cavaglia, V. Alfaro and A. T. Filippov, Int. J. Mod. Phys. A 10, 611 (1995).

[8] N. A. Lemos, J. Math. Phys. 37, 1449 (1996);

[9] N. A. Lemos, G. A. Monerat, Gen. Rel. Grav. 35, 423 (2003); J. Acacio de Barros and N. Pinto-Neto, Int. J. Mod. Phys. D 7, 201 (1998); J. Acacio de Barros, N. Pinto-Neto and M. A. Sagioro-Leal, Phys. Lett. A 241, 229 (1998).
[10] F. G. Alvarenga, J. C. Fabris, N. A. Lemos, G. A. Monerat, Gen. Rel. Grav. 34, 651 (2002).

[11] S. Carlip, Phys. Rev. Lett. 794071 (1997).

[12] M. Anderson, S. Carlip, J.G. Ratcliffe, S. Surya and S.T. Tschantz, Class. Quant. Grav. 21 729, (2004).

[13] Schutz, B. F., Phys. Rev. D 2, 2762, (1970); Schutz, B. F., Phys. Rev. D 4, 3359, (1971).

[14] P. A. M. Dirac, Can. J. Math. 2, 129 (1950); Proc. Roy. Soc. London A bf 249, 326 and 333 (1958); Phys. Rev. 114, 924 (1959).

[15] S. C. Chhajlany and V. N. Malnev, Phys. Rev. A, vol 42, No. 5 , 3111 p., (1990); S. C. Chhajlany, D. A. Letov and V. N. Malnev, J. Phys. A: Math. Gen. 24, 2731 (1991).

[16] H. Everett, III, Rev. Mod. Phys. 29, 454 (1957).

[17] E. M. Lifshitz, L. D. Landau, Quantum Mechanics: NonRelativistic Theory, Volume 3, Third Edition (Quantum Mechanics), (Butterworth-Heinemann, Oxford, 2003).

[18] G. A. Monerat, E. V. Corrêa Silva, G. Oliveira-Neto, L. G. Ferreira Filho and N. A. Lemos, gr-qc/0508086. 